\title{
Changing from intensive anticoagulation to treatment with aspirin alone for coronary stents: the experience of one centre in the United Kingdom
}

\author{
N G Stephens, P F Ludman, M C Petch, P M Schofield, L M Shapiro
}

\begin{abstract}
Objective-To investigate whether an elective change in the anticoagulation protocol for patients with coronary stents affected clinical outcomes and length of hospital stay.

Design-Retrospective observational study of a consecutive series of patients treated with coronary stents over an 18 month period from April 1994 to October 1995.

Background-Intensive anticoagulation regimens are used in many UK centres to reduce the risk of coronary stent thrombosis. Recent data have called into question the necessity for full anticoagulation and favourable results have been reported with antiplatelet agents alone. The results from a tertiary referral centre were investigated during a period where an elective change in policy was made: an initial $\mathbf{7 0}$ patients were treated intensively with intravenous heparin and with warfarin and aspirin; subsequently 94 were treated with aspirin and deployment of a high pressure balloon only.
\end{abstract}

Methods-Review of case notes, angiograms, and a database of intervention procedures and telephone interview. Classic epidemiological techniques, as well as linear regression and logistic regression, were used to model the outcomes of major procedural complications and length of hospital stay.

Patients-164 patients treated with 196 coronary stents.

Results-There were 22 (13.4\%) major complications (coronary bypass grafting 11 , subacute thrombosis 6 , tamponade 2 , myocardial infarction 1 , death 2 ). With logistic regression, the risk of major complication was shown not to be affected by anticoagulation (relative risk (RR) 1.03; $P=0.97$ ). Significant determinants of risk included acute vessel closure as an indication for stenting $(R R=80.6$; $P<0.001$ ) and sex (male: female $R R=$ $0.19 ; \quad P=0.02$ ). The median length of stay (LOS) was 5 days (1-45). Use of a linear regression model showed that anticoagulation added 4.5 days and a major complication added a further 4.5 days to a baseline length of stay of 3.2 days $\left(R^{2}=\right.$ 0.32; $P<0.001$ ).

Conclusion-This is a report of coronary stenting as part of usual clinical practice in one British tertiary referral centre. In this experience, treatment with aspirin alone is probably as safe as intensive anticoagulation, and has the benefit of reducing length of stay by more than $50 \%$ to $3 \cdot 2$ days in an uncomplicated case.

(Heart 1996;76:238-242)

Keywords: stent; aspirin; anticoagulation; thrombosis

Since the first report of coronary stent implantation in man, ${ }^{1}$ the technique has become widely adopted. It has the virtue of reducing the incidence of restenosis in selected cases, ${ }^{23}$ and reducing the need for urgent coronary artery bypass grafting (CABG) to rescue patients with occlusive dissection after percutaneous transluminal coronary angioplasty (PTCA). ${ }^{4}$ The weakness of stent implantation is the complication of subacute thrombosis (SAT). This occurs at an unpredictable period-between two days and three weeks after the procedure. ${ }^{5}$ SAT may therefore cause myocardial infarction or death after hospital discharge-when rescue CABG is not rapidly available. There are no adequate randomised controlled trials of treatments or treatment strategies that are designed to minimise this risk. The first report of experience with the slotted steel Palmaz-Schatz stent showed a $15 \%$ incidence of SAT in the initial cohort of 39 patients treated with antiplatelet agents alone and a $0.6 \%$ incidence in the subsequent 174 treated with an intense regimen of intravenous heparin, intravenous dextran, aspirin, dipyridamole, and warfarin. ${ }^{6}$ These data have influenced the widespread adoption of similar regimens (generally without dextran or dipyridamole) which remain current practice in most cardiac centres in the UK (unpublished data from the second Leeds Advanced Coronary Stent Symposium, October 1995).

Aggressive anticoagulation carries considerable penalties: a prolonged hospital stay (typically 6-9 days) and a greatly increased risk of vascular access complications and haemorrhage (a reported incidence of $7-14 \%) .{ }^{23}$ Recent studies from a single centre in Italy ${ }^{7}$ and from a French multicentre registry ${ }^{8}$ reexamined the necessity for anticoagulation, and reported subacute thrombosis (SAT) rates of $1-1 \cdot 6 \%$ when the antiplatelet agents aspirin and ticlopidine alone were used. These data suggest that factors such as experience, lesion selection, and balloon size and pressure are the important determinants of SAT-contrary to the early conclusions of the pioneers of the 
technique. On the basis of the reported experience with antiplatelet agents alone, we elected to change from intensive anticoagulation to treatment with aspirin alone in March 1995. This report is based on our analysis of major complications and change in length of hospital stay in 164 patients over an 18 month period up to October 1995. Seventy patients were treated with a conventional "aggressive" anticoagulation regimen and 94 with aspirin alone.

\section{Patients and methods PATIENTS}

We examined the case records and angiograms of 164 consecutive patients treated with coronary stents in Papworth Hospital between March 1994 and October 1995. They were identified from three sources: the cardiac catheterisation laboratory $\log$, a database of all intervention procedures, and the hospital administration system. There was $100 \%$ ascertainment of all intervention cases where stents were implanted in the coronary artery - that is, this analysis is by "intention to stent". Data were collected on age, sex, clinical presentation, lesion(s) treated, equipment used, and drug regimen. The nominal diameter of the deployed balloon at the maximum pressure used (from the manufacturer's quoted compliance) was taken as the final diameter of the stented segment. Forty three advanced vascular engineering (AVE) type 1 steel stents, 75 Palmaz-Schatz slotted steel stents, and 26 Wiktor tantalum stents were used over the study period. There were no predetermined indications for each type, these were chosen at the discretion of the five operators in the study. Quantitative angiography was not used to determine reference vessel diameter-thus, the "balloon-artery ratio" was not calculated. Unstable angina was defined as chest pain that required hospital admission and in-patient revascularisation. Intravascular ultrasound (IVUS) was not used to guide stent deployment. Thirty one patients were readmitted or had clinical events during their hospital stay after the procedure. An additional 114 patients who had not been readmitted to hospital were interviewed by telephone to determine whether any had experienced symptoms compatible with subacute stent thrombosis after hospital discharge.

\section{OUTCOME MEASURES}

We examined two outcomes: major procedural complications and length of hospital stay. Complications were classified as:

(1) Cardiac death (at any time)

(2) Myocardial infarction (MI) within one month (defined by modified WHO criteria ${ }^{9}$ )

(3) $\mathrm{CABG}$ for vessel closure within one month of the procedure

(4) Subacute stent thrombosis (this required angiographic proof of target vessel closure within one month )

(5) Cardiac tamponade. This life-threatening adverse outcome was included in the analysis because of its severity, but was not judged to be mechanistically related to subacute thrombosis.

\section{ANTICOAGULATION}

All patients were taking $75 \mathrm{mg}$ aspirin/day at baseline. Those treated with intensive anticoagulation received an initial $15000 \mathrm{U}$ of heparin during the catheter laboratory procedure, with further $3000 \mathrm{U}$ boluses to maintain the activated clotting time (ACT) at more than 350 seconds. After the procedure, no further heparin was given. The ACT was measured every 15 minutes on the ward, with removal of the sheaths once the value was less than $200 \mathrm{~s}$. After 30 minutes haemostasis without compression, heparin was restarted, with a target ACT of 190-220 s. Warfarin was given on the evening of the procedure, with a target international normalised ratio (INR) of 2.5-3.5. The heparin was continued for 24 hours after the INR had reached $2 \cdot 5$.

After the change in protocol, patients treated with aspirin alone received heparin during the procedure to maintain the ACT $>350$ s, and none thereafter. Most patients received $75 \mathrm{mg}$ of aspirin; $25 \mathrm{had} 300 \mathrm{mg}$ at the time of the procedure and $300 \mathrm{mg} /$ day thereafter. Ticlopidine was not used because of concerns about the risk of bone marrow toxicity and because it was available only on a named patient basis. Four cases were treated with the full anticoagulation protocol after the change in policy; in two cases this was for a suboptimal result with stent outflow dissection and two other patients were taking warfarin for another indication.

\section{STATISTICAL ANALYSIS}

Baseline variables are reported as means with standard deviation (for normally distributed variables), medians with range (for other continuous variables), and percentages (categorical variables). The Shapiro-Wilks statistic was used to test the departure of the distribution of continuous variables from normality. Differences between groups in baseline variables and the influence of baseline variables on the outcome of major complications were tested with Pearson's $\chi^{2}$ (with odds ratios calculated with the approximation of Woolf), the Mantel-Haenszel test, and Student's t test as appropriate. Logistic regression was used for multivariate analysis, with 11 variables entered in a single, "forced entry" step. Continuous variables were dichotomised around the median to maximise the power of the analyses. Stepwise linear regression was used to model the effect of all potential explanatory variables on length of stay. Variables were entered backwards into the model, with threshold values of the $F$ statistic of 4 for entry and 2 for remaining in the model. A two tailed $P$ value of $<0.05$ was considered significant in all analyses. Odds ratios and coefficients are quoted with $95 \%$ confidence intervals (CI). Stata 4.0 software was used for all analyses. ${ }^{10}$

\section{Results}

The figure gives a graphical representation in the form of a oneway scatterplot of the change in policy over time. The timing of individual cases over the duration of the study is shown, with the abrupt transition in the management 
Change in anticoagulation protocol over the study period (days).
0

| ||| |||||| || | || || || | || || || ||||| |||||||

562

Cases treated with aspirin alone policy and an increasing frequency of stent implantation with time. The four cases treated with anticoagulation after the transition are shown. Three had an uncomplicated course, and one had cardiac tamponade requiring emergency drainage.

The baseline characteristics of the patients and procedures, by anticoagulation regimen, are shown in table 1 . There are differences between the groups which reflect changes in technique and indication for stenting - and which underline the necessity for multivariate analysis to isolate the influence of the anticoagulation regimen itself on outcomes. One major change in technique coincident with the change to aspirin alone was the use of noncompliant high pressure balloons for stent deployment, with a target deploying balloon pressure of 16 bar. Use of the Palmaz-Schatz stent increased over the 18 month period, with the indication for the Wiktor stent changing from a first line choice to a "niche" role for lesions on acute bends or with major side branches. With increasing experience there was a greater elective use of stents (that is, planned before the procedure, rather than used in the event of dissection) and a reduction in enthusiasm for stenting lesions of saphenous vein grafts.

Table 1 Characteristics of patients and lesions stented with full anticoagulation or aspirin alone

\begin{tabular}{lccl}
\hline & $\begin{array}{c}\text { Anticoagulated } \\
(n=70)\end{array}$ & $\begin{array}{c}\text { Aspirin alone } \\
(n=94)\end{array}$ & $P$ \\
\hline Age: mean (SD) & $59 \cdot 5(8)$ & $59 \cdot 1(9)$ & $0 \cdot 83$ \\
Male (\%) & $59(84 \%)$ & $74(79 \%)$ & $0 \cdot 47$ \\
Unstable angina (\%) & $22(31 \%)$ & $26(28 \%)$ & $0 \cdot 60$ \\
Number of stents (median (range)) & $1(1-3)$ & $1(1-4)$ & $0 \cdot 80$ \\
Stented length (mm) (median (range) & $15(8-48)$ & $15(8-32)$ & $0 \cdot 49$ \\
Maximum pressure (bar) (median (range)) & $10(6-19)$ & $16(6-20)$ & $<0.001$ \\
Final lesion diameter (mm) (median (range)) & $3 \cdot 5(3-6)$ & $3 \cdot 4(2 \cdot 5-4 \cdot 5)$ & $0 \cdot 02$ \\
Stent: & $22(31 \%)$ & $21(22 \%)$ & \\
AVE & $27(39 \%)$ & $68(72 \%)$ & $<0 \cdot 001$ \\
Palmaz-Schatz & $21(30 \%)$ & $5(5 \%)$ & \\
Wiktor & $20(29 \%)$ & $40(43 \%)$ & \\
Vessel: & $11(16 \%)$ & $19(20 \%)$ & \\
LAD & 0 & $1(1 \%)$ & $0 \cdot 04$ \\
LCx & 0 & $2(2 \%)$ & \\
LIMA & $24(34 \%)$ & $26(28 \%)$ & \\
LMS & $15(21 \%)$ & $6(6 \%)$ & \\
RCA & $4(6 \%)$ & $5(4 \%)$ & \\
SVG & $21(30 \%)$ & $30(32 \%)$ & \\
Indication: & $16(23 \%)$ & $29(31 \%)$ & $0 \cdot 05$ \\
Acute closure & $8(9 \%)$ & \\
Dissection & $2(3 \%)$ & $19(20 \%)$ & \\
Elective & $14(20 \%)$ & $4(4 \%)$ & \\
Chronic occlusion & $13(19 \%)$ & & \\
Restenosis & &
\end{tabular}

LAD, left anterior descending; LCx, left circumflex; LIMA, left internal mammary artery; LMS, left main stem; RCA, right coronary artery; SVG, saphenous vein graft.
There were $22(13.4 \%)$ major complica-

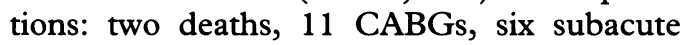
thromboses, two early post-procedural tamponades, and one MI. One additional patient experienced a sudden recurrence of angina at one week after the procedure, which resolved spontaneously without subsequent ECG change. The patient was not admitted to hospital. This case of possible SAT is not included in the analysis. The major complications are tabulated by antiocoagulation regimen in table 2. The overall proportion of adverse events was similar in each group (11/70 (16\%) for those given intensive anticoagulation and $11 / 94(12 \%)$ aspirin alone; $\mathrm{P}=$ $0 \cdot 78$ ). Both deaths occurred in the aspirintreated group. Of these, necropsy in one case showed a patent stent 24 hours after the procedure, with arrhythmia being the most likely cause of death. There was evidence of chronic stent occlusion in the other patient, who died suddenly at three months. It could not be determined histologically whether this was a case of organised thrombus or restenosis. Both cases of cardiac tamponade occurred in patients treated with intensive anticoagulation. One was judged to have been caused by right ventricular perforation by a temporary pacing electrode and one was caused by coronary artery perforation by the angioplasty guide wire. All events are regarded as a single combined endpoint to increase the power of the analysis and because all are cardiac complications directly related to the interventional procedure. The high rate of CABG reflects the inclusion of cases where coronary stents were deployed to relieve ischaemia and as a bridge to CABG after failed PTCA. They are included here, but in none of the cases can they be regarded as an unanticipated complication of stent deployment.

Table 3 details the analysis of the relation of the baseline patient and lesion variables to the

Table 2 Major complications by anticoagulation regimen

\begin{tabular}{lll}
\hline Complication & $\begin{array}{l}\text { Full anticoagulation } \\
(n=70)\end{array}$ & $\begin{array}{l}\text { Aspirin alone } \\
(n=94)\end{array}$ \\
\hline Death & 0 & 2 \\
CABG & 6 & 5 \\
MI & 0 & 1 \\
SAT & 3 & 3 \\
Tamponade & 2 & 0
\end{tabular}

CABG, coronary artery bypass grafting; MI, myocardial infarction; SAT, subacute thrombosis. 
Table 3 Odds ratios (OR) of major complication for 11 variables

\begin{tabular}{|c|c|c|c|c|}
\hline & $\begin{array}{l}\text { Univariate } \\
\text { OR }(95 \% C I)\end{array}$ & $P$ & $\begin{array}{l}\text { Multivariate } \\
\text { OR }(95 \% \text { CI })\end{array}$ & $P$ \\
\hline Anticoagulation $v$ aspirin alone & $1.14(0.46$ to 2.82$)$ & $0 \cdot 78$ & $1.03(0.25$ to 4.23$)$ & 0.97 \\
\hline Acute closure $v$ other indication & $26 \cdot 3(4 \cdot 13$ to 167$)$ & $<0.001$ & $80 \cdot 6(8 \cdot 10$ to 801$)$ & $<0.001$ \\
\hline Palmaz-Schatz $v$ other stent & $1.66(0.63$ to 4.34$)$ & $0 \cdot 30$ & $6.31(1.45$ to $27 \cdot 4)$ & 0.01 \\
\hline Male $v$ female & $0.44(0.16$ to 1.20$)$ & $0 \cdot 10$ & $0.19(0.05$ to 0.78$)$ & 0.02 \\
\hline Age & $0.53(0.21$ to 1.34$)$ & $0 \cdot 17$ & $0.36(0.11$ to 1.25$)$ & $0 \cdot 11$ \\
\hline Number of stents & $2 \cdot 75(1.11$ to $6 \cdot 84)$ & 0.03 & $1.94(0.82$ to 4.55$)$ & $0 \cdot 13$ \\
\hline Maximum pressure used & $0.68(0.28$ to 1.69$)$ & 0.41 & $0.43(0.09$ to 1.95$)$ & $0 \cdot 27$ \\
\hline Unstable angina & $1 \cdot 15(0.45$ to 3.04$)$ & $0 \cdot 78$ & $1.83(0.56$ to 5.91$)$ & $0 \cdot 31$ \\
\hline Final lesion diameter & $0.64(0.25$ to 1.60$)$ & 0.33 & $1.49(0.43$ to 5.22$)$ & 0.53 \\
\hline LAD $v$ other vessel & $1.24(0.35$ to 4.32$)$ & $0 \cdot 74$ & $1.24(0.35$ to 4.32$)$ & 0.74 \\
\hline Length of stented segment & $1.22(0.33$ to 4.50$)$ & $0 \cdot 31$ & $0.97(0.17$ to 5.50$)$ & 0.97 \\
\hline
\end{tabular}

Continuous variables are dichotomised around a median value and the two groups compared.

risk of major complications. Anticoagulation was not associated with an increased risk: the OR was near unity $(1.03 ; P=0.97)$. The most powerful predictor of complication was stent implantation to bail out acute vessel closure, with $O R=81$ in the regression analysis $(P<0.001)$. Six of the eight cases of acute closure resulted in a major complication (CABG in four, one SAT, and one tamponade). Other independent predictors of a major complication were use of a Palmaz-Schatz stent and female sex. There were also trends towards independent effects of high balloon pressure on risk reduction (OR 0.43 ) and increasing procedural risk with the number of stents deployed per case (OR 1.94 for each additional stent used).

There were four cases of femoral artery pseudoaneurysm which required surgical repair-three $(4 \%)$ in anticoagulated patients and one $(1 \%)$ in a patient taking aspirin alone (OR 2.25; $\mathrm{P}=0.50$ ).

Using stepwise linear regression, we derived a model which accounted for $32 \%$ of the variation in length of stay (LOS):

LOS $=3.24+4.54$ (anticoag) +4.51 (major complication)

Each independent variable in the model is coded 1 or 0 . Thus, the baseline LOS in an uncomplicated case treated with aspirin alone is 3.2 days ( $95 \%$ CI $2 \cdot 4$ to $4 \cdot 1$ ), with 4.5 (3.3 to $5 \cdot 8)$ days added if the patient was anticoagulated and $4.5(2.7$ to $6 \cdot 3)$ added if the procedure was associated with a major complication.

\section{Discussion}

This is an observational study of a change in practice with coronary stents in one tertiary referral centre in the UK. It provides further evidence that early outcomes of coronary stenting are as good with aspirin alone as they are with an intensive anticoagulation regimen. This outcome was obtained without the potential benefit of ticlopidine or IVUS guided stent deployment. Although the "best guess" is that the risks are similar for each policy, the confidence intervals on the odds ratio estimate are wide $(0.25$ to $4 \cdot 21)$, reflecting the lack of power in the study. Indeed, over 1000 patients would be required to demonstrate a reduction in the complication rate from $12 \%$ to $6 \%$ with any treatment (with $90 \%$ power and $\alpha=$ $0.05)$. Thus these data provide supporting evidence that may increase the confidence of other centres considering a change in anticoagulation regimen. This is not, of course, a randomised trial of two treatments, and our conclusions are reached using epidemiological modelling methods with their traditional caveats related to association versus causation and confounding. For example, although use of a Palmaz-Schatz stent was associated with an increased risk of complication in this study, this may be because operators prefer to use this device with "high risk" cases rather than because the stent itself has adverse effects. Our finding that women were at higher risk in this series (independent of arterial size and age) adds to the findings of adverse outcomes in PTCA and CABG, which remain largely unexplained. ${ }^{11-13}$

The equivocal benefit of changing to aspirin alone would be of little interest if there were no other tangible benefits. However, this change in policy had the beneficial effect of more than halving hospital stay to $3 \cdot 2$ days in an uncomplicated case, with the implied considerable reductions in morbidity and hospital costs.

We conclude that stent implantation with aspirin alone is probably as safe as intensive anticoagulation, and brings considerable benefits in reducing length of hospital stay.

1 Sigwart U, Puel J, Mirkovitch V, Joffre F, Kappenberger L. Intravascular stents to prevent occlusion and restenosis after transluminal angioplasty. $N$ Engl f $\mathrm{Med}$ 1987;316: 701-6.

2 Serruys PW, de Jaegere P, Kiemeneij F, Macaya C, Rutsch W, Heyndrickx G, et al. A comparison of balloonexpandable-stent implantation with balloon angioplasty in patients with coronary artery disease. Benestent Study Group. N Engl f Med 1994;331:489-95.

3 Fischman DL, Leon MB, Baim DS, Schatz RA, Savage MP, Penn I, et al. A randomized comparison of coronarystent placement and balloon angioplasty in the treatment of coronary artery disease. Stent Restenosis Study Investigators. N Engl F Med 1994;331:496-501.

4 Kuntz RE, Piana R, Pomerantz RM, Carrozza J, Fishman $\mathrm{R}$, Mansour $\mathrm{M}$, et al. Changing incidence and management of abrupt closure following coronary intervention in the new device era. Cath Cardiovasc Diag 1992;27: $183-90$.

5 Savage MP, Fischman DL, Schatz RA, Teirstein PS, Leon $\mathrm{MB}$, Baim $\mathrm{D}$, et al. Long-term angiographic and clinical outcome after implantation of a balloon-expandable stent in the native coronary circulation. Palmaz-Schatz Stent Study Group. $¥ A C C$ 1994;24:1207-12.

6 Schatz RA, Goldberg S, Leon M, Baim D, Hirshfeld J, Cleman $M$, et al. Clinical experience with the PalmazSchatz coronary stent. $\mathcal{F A C C} 1991 ; 17(6$ suppl $\mathrm{B})$ : 155B-9B.

7 Colombo A, Hall P, Nakamura S, Almagor Y, Maiello L, Martini G, et al. Intracoronary stenting without anticoagulation accomplished with intravascular ultrasound guidulation accomplished with intravascular
ance. Circulation 1995;91:1676-88.

8 Morice MC, Breton C, Bunouf P, Cattan S, Eltchaninoff H, Henry M, et al. Coronary stenting without anticoagu$\mathrm{H}$, Henry $\mathrm{M}$, et al. Coronary stenting without anticoagu-
lant, without intravascular ultrasound. Reults of the lant, without intravascular ultrasound. Reu
French registry. Circulation 1995;92:I-796.

9 Palomaki P, Miettinen H, Mustaniemi H, Lehto S, Pyorala $\mathrm{K}$, Mahonen $\mathrm{M}$, et al. Diagnosis of acute myocardial infarction by MONICA and FINMONICA diagnostic 
criteria in comparison with hospital discharge diagnosis. f Clin Epidemiol 1994;47:659-66.

10 StataCorp. Stata Statistical Software: Release 4.0. College Station: Texas USA: Stata Corporation, 1995.

11 Bell MR, Grill DE, Garratt KN, Berger PB, Gersh BJ, Holmes DR Jr. Long-term outcome of women compared with men after successful coronary angioplasty. Circulation 1995;91:2876-81.
12 Jaglal SB, Tu JV, Naylor CD. Higher in-hospital mortality in female patients following coronary artery bypass surgery: a population-based study. Provincial Adult Cardiac Care Network of Ontario. Clin Invest Med 1995;18:99-107.

13 Barbir M, Lazem F, Ilsley C, Mitchell A, Khaghani A, Yacoub $M$. Coronary artery surgery in women compared with men: analysis of coronary risk factors and in-hospital mortality in a single centre. Br Heart $\mathcal{F} 1994 ; 71: 408-12$.

\section{IMAGES IN CARDIOLOGY}

\section{Ball thrombus in the left ventricle}

This echocardiogram was performed on an 84 year old woman admitted with left ventricular failure. She had suffered an anterior myocardial infarction 15 years previously, and was known to have chronic lymphatic leukaemia. She had not received antiplatelet or anticoagulant treatment in the past.

The cross sectional echocardiogram (apical four chamber view) demonstrates a large ball thrombus (arrow) floating freely in the left ventricle. There is evidence of a previous myocardial infarction: the inter-ventricular septum is aneurysmal and the left ventricle is globally dilated and poorly contractile. The aortic valve is calcified and stenosed, preventing the thrombus from leaving the ventricular cavity. Indeed on several occasions the thrombus was seen to "bounce off" the aortic valve.

Although the incidence of left ventricular thrombus after myocardial infarction is about $30 \%$, the finding of thrombus completely free in the left ventricle is rare. There are some reports of surgical removal of such thrombus. However, we believed that this was inappropriate in this frail patient. She died four months later.

SIMON WOLDMAN JAVIER GUELL-PERIS

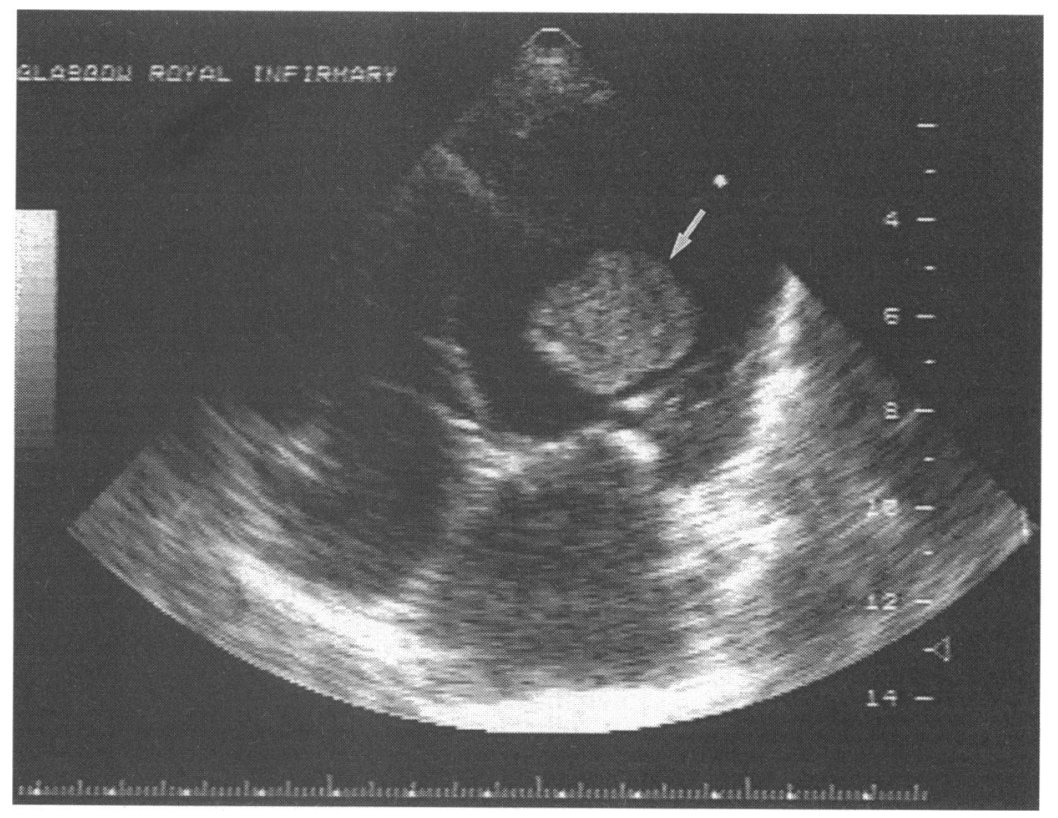

